\title{
Le collier du chancelier de l'université de Strasbourg
}

Daniel Bornemann

\section{(2) OpenEdition}

1 Journals

\section{Édition électronique}

URL : https://journals.openedition.org/rbnu/3239

DOI : 10.4000/rbnu.3239

ISSN : 2679-6104

\section{Éditeur}

Bibliothèque nationale et universitaire de Strasbourg

\section{Édition imprimée}

Date de publication : 1 novembre 2011

Pagination : 86-89

ISSN : 2109-2761

\section{Référence électronique}

Daniel Bornemann, «Le collier du chancelier de l'université de Strasbourg », La Revue de la BNU [En ligne], 4 | 2011, mis en ligne le 01 novembre 2011, consulté le 08 août 2021. URL : http:// journals.openedition.org/rbnu/3239; DOI : https://doi.org/10.4000/rbnu.3239

\section{(c) (†)(2)}

La Revue de la BNU est mise à disposition selon les termes de la Licence Creative Commons Attribution - Pas d'Utilisation Commerciale - Partage dans les Mêmes Conditions 4.0 International. 


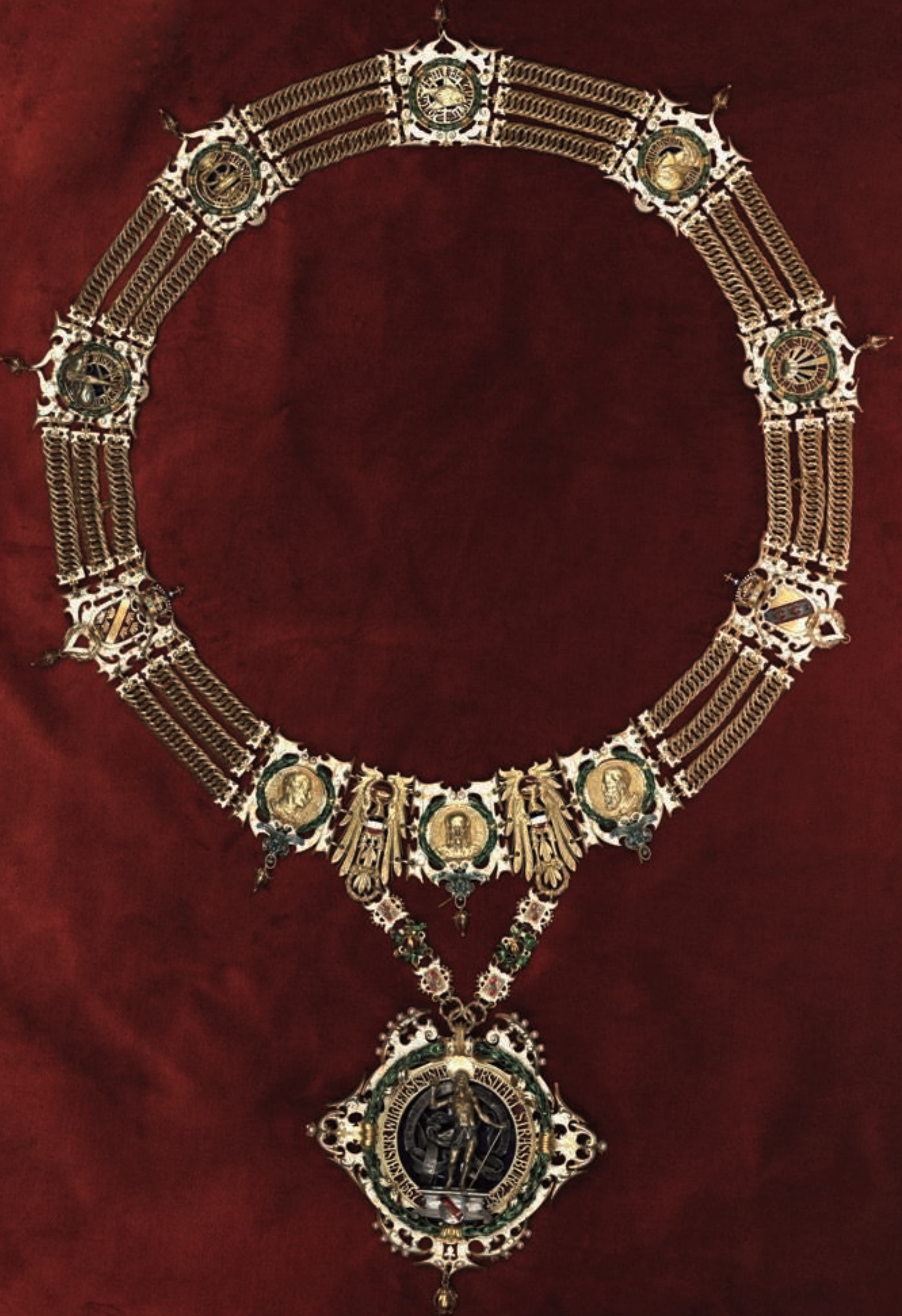




\section{LE COLLIER DU CHANGELIER de l'université de Strasbourg}

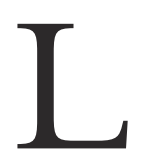

a BNU a en dépôt depuis le lendemain de la Seconde Guerre mondiale la " chaîne du recteur ", autrement dit le collier d'armes du recteur et chancelier des universités de Strasbourg. Elle a été inventoriée sous le numéro 14308 le 8 juillet 1950. Cette pièce de grande allure a été dessinée par l'artiste strasbourgeois (munichois de naissance) Anton Seder, en 1897, dans un style néo-renaissance, le même que celui des bâtiments de l'université impériale, un style que les orfèvres maniéristes rhénans illustrèrent particulièrement aux $16^{\mathrm{e}}$ et $17^{\mathrm{e}}$ siècles. Cet artiste et professeur d'arts appliqués était depuis 1889 directeur de l'Ecole des arts décoratifs de Strasbourg. D’après son projet, la chaîne fut réalisée par le ciseleur et joaillier Walther Eberbach (1866-1944). C'était une commande de l'empereur Guillaume II pour fêter le vingt-cinquième anniversaire de la création de son université. Ce bijou était sans doute aussi destiné à compléter le décorum de l'institution et à affirmer encore un peu plus la mainmise allemande sur la culture alsacienne, par l'exhibition de la richesse de la dotation impériale. Les maires de Strasbourg et de Metz reçurent également chacun un collier d'armes (Ehrenkette) dans le même style. Ce collier a été réalisé en or, et porte abondance de pierreries, parmi lesquelles des diamants, des rubis, des turquoises, également des perles fines en grand nombre, ainsi que de nombreux symboles fi- nement ciselés. Certains éléments de la chaîne sont émaillés en blanc, d'autres en diverses couleurs. Une grande figure du Christ en ronde-bosse, sortant de son tombeau, bénissant, et qui semble s'appuyer sur une canne très peu canonique, occupe le médaillon principal. La canne de Jésus ? C'était depuis Constantin un étendard de l'Église, un « labarum " dont la partie supérieure, à partir de la main du Christ, manque, et ce depuis le printemps 1941. Un phylactère précise que ce Christ est le sceau de l'académie de Strasbourg (" Sig. Academiae Argentinae "). Des médailles de la même époque montrent en effet ce même motif, avec la même légende. Le programme symbolique de cette chaîne est ensuite, en zone pectorale, constitué de deux aigles impériales, d'écus aux couleurs de la Prusse, de trois médailles à l'effigie des trois empereurs Hohenzollern successifs ; les armes de l'Alsace et de la Lorraine, en émaux fins, et les symboles des cinq facultés : jurisprudence, médecine, théologie, philosophie, sciences naturelles, se répartissent autour du cou. Le blason de Strasbourg est, lui, figuré sous les pieds du Christ. Le poids de la chaîne est de 1715 grammes. L'inscription entourant le Christ est " 1567 Kaiser Wilhelms Universitaet Strassburg 1872 ". Le pouvoir en place usa ainsi du style de la Renaissance strasbourgeoise dans la démarche d'annexion. La chaîne est moyennement bien conservée : certaines pendeloques sont manquantes, et comme nous l'avons 
signalé, un attribut du Christ manque également. Alphonse Irjud révèle quelques détails intéressants sur les tribulations de ce bijou durant la Seconde Guerre mondiale. A l'instar des collections de l'université, il fut évacué à Clermont-Ferrand. Mais peu après l'armistice de 1940, des émissaires allemands vinrent tenter de récupérer les biens de l'université afin de créer la "Reichsuniversität ". La récupération du collier faisait partie des objectifs de leur mission. Une résistance administrative éphémère n'empêcha pas longtemps sa remise aux mains des nazis, mais une " croix à deux traverses " se détacha à ce moment-là du médaillon central. On y avait presque vu une croix de Lorraine! C'est donc que le drapeau avait déjà disparu et qu'il restait la hampe supérieure et sans doute un autre élément d'appui horizontal. En fait il s'agissait d'un étendard portant une croix et gonflé par un courant d'air. Le commissaire chargé de la récupération des biens décrit alors la chaîne comme " endommagée à plusieurs endroits par des manipulations négligentes ". Un bijoutier de Karlsruhe la reçoit afin d'y remédier, et adresse sa facture au président du conseil d'administration de l'université, qui refuse de l'acquitter. Ces frais étaient-ils relatifs à la réfection de la chaîne ou à la fabrication d'un étui ? Il ne semble pas que des réparations aient été faites néanmoins. Les discussions semblent avoir été vives et portent pour beaucoup sur le bel étui tout neuf. En effet la chaîne n'en avait pas au moment où elle fut remise au commissaire nazi : elle était " enveloppée dans du papier journal et des serviettes ". Grandeurs et misères des fastes impériaux ! On ne sait pas quel fut le fin mot de cette querelle. Toujours est-il qu'à la Libération le collier fut retrouvé avec son étui au fond d'un tiroir du Palais universitaire.

\section{Pour en savoir plus}

- Strassburger historische Schmuck Ausstellung im alten Rohan-Schloss. Exposition de bijoux anciens et modernes au Château des Rohan, Sept.-Okt. 1904. Strasbourg : Dusch, 1904. $\mathrm{N}^{\circ}$ 2, p. 5, non reproduit.

— Geschichte des Gold- \& Silberschmuckes nach Originalen der Strassburger historischen Schmuckausstellung von 1904 / Robert Forrer. Strasbourg : Ludolf Beust, 1905. N²69, p. 51.

- Une curieuse chasse au trésor : la récupération des biens culturels / Alphonse Irjud, in Saisons d'Alsace, $\mathrm{n}^{\circ}$ 114, 1991, p. 79-84.

- Impressions d'Europe. - Strasbourg : BNU, 2003. Catalogue $\mathrm{n}^{\circ} 218,4$, p. 74.

\section{Daniel Bornemann}




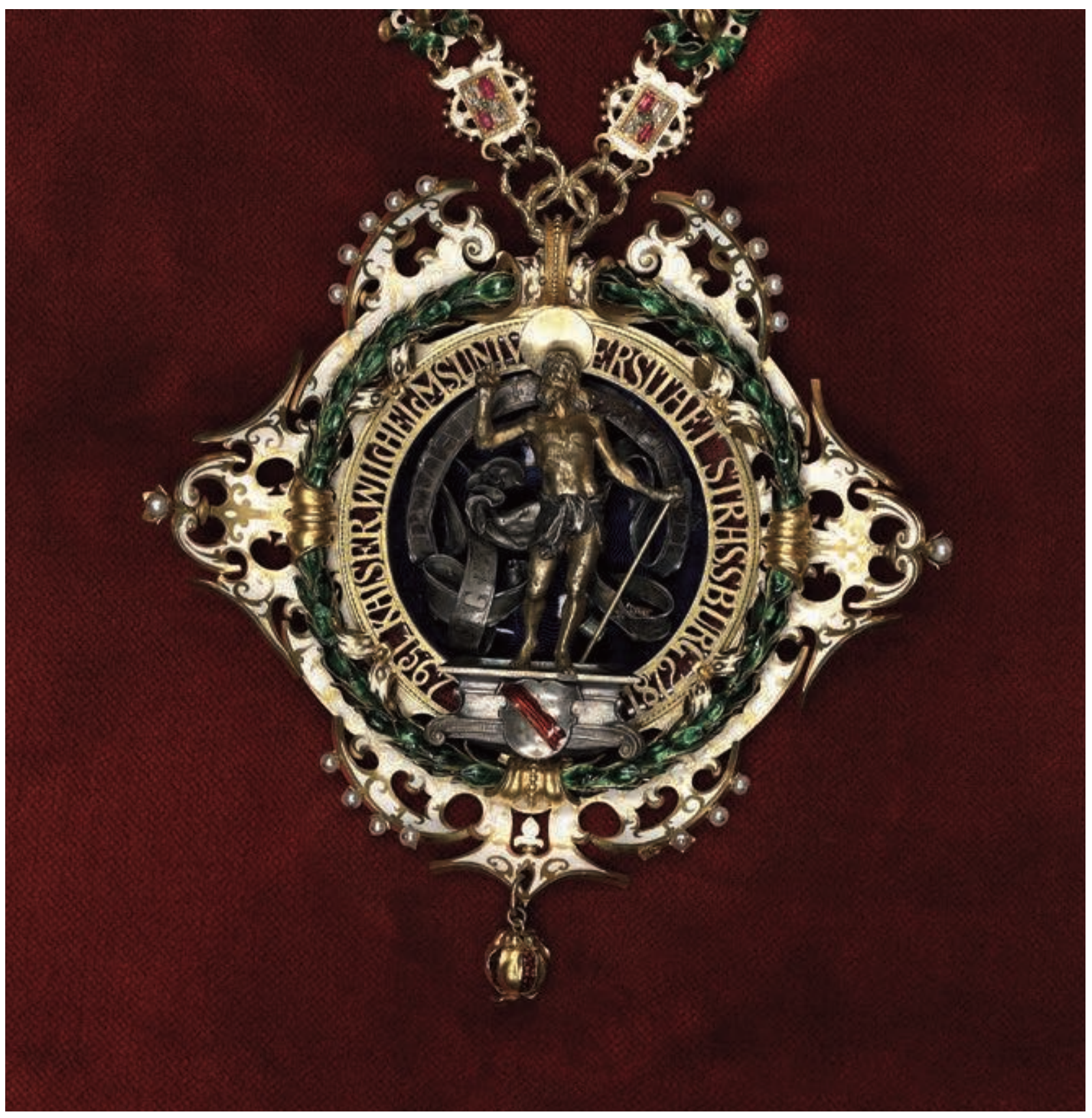

